Agron. Mesoam. 29(1):53-61. Enero-abril, 2018

ISSN 2215-3608, doi:10.15517/ma.v29i1.27114

http://www.revistas.ucr.ac.cr/index.php/agromeso

\title{
Identificación molecular de aislamientos de Fusarium asociados a maracuyá en el Valle del Cauca, Colombia ${ }^{1}$
}

\section{Molecular identification of Fusarium isolates associated with passion fruit in five locations from Valle del Cauca, Colombia}

\author{
Edwin David Henao-Henao ${ }^{2,4}$, Carlos Alberto Hernández-Medina², Claudia Salazar-González ${ }^{3}$, \\ Martha Lucia Velasco-Belalcazar², Eyder Daniel Gómez-López²
}

\begin{abstract}
Resumen
La producción de maracuyá en Colombia ha mostrado grandes avances; sin embargo, las enfermedades continúan siendo un factor limitante en la producción del mismo. Entre los agentes patógenos que dificultan el establecimiento y desarrollo del cultivo, se encuentran algunas especies del género Fusarium, consideradas de importancia económica por las pérdidas que generan. El objetivo de este trabajo fue identificar molecularmente las poblaciones de Fusarium presentes en maracuyá (Passiflora edulis). Entre enero y diciembre de 2015 se realizó el aislamiento de 35 muestras de diferentes órganos afectados de la planta y provenientes de localidades ubicadas en cinco municipios del Valle del Cauca, Colombia. Se seleccionaron y purificaron ocho aislamientos pertenecientes al género de interés para realizar la extracción de ADN y amplificación de la región TEF 1 alpha. Los productos de PCR fueron secuenciados y comparados con las bases de datos del NCBI y del Fusarium ID. Los aislamientos pertenecían a: Fusarium incarnatum, Fusarium proliferatum y Fusarium solani, la primera especie fue la de mayor ocurrencia en las muestras evaluadas. En el análisis de las relaciones filogenéticas realizado mediante el programa MEGA 6, se usó el coeficiente de similitud del vecino más cercano, lo que permitió observar variabilidad entre los aislamientos evaluados.
\end{abstract}

Palabras clave: fusariosis, Passiflora edulis, enfermedades fungosas, variación genética.

\begin{abstract}
The passion fruit's production in Colombia has shown great advances; however, phytosanitary diseases remain as issues in passion fruit production. This includes the pathogens that hinder the establishment and development of the crop, such as some species of the genus Fusarium, pathogen considered as an important one because of the economic losses that it generates. The objective of this work was to molecularly identificate the populations of Fusarium associated with passion fruit (Passiflora edulis). Between January and December 2015, an isolation of 35 samples based on different affected organs of the plant and from localities found in five municipalities was performed in Valle del Cauca, Colombia. Eight isolates were selected and purified in order to perform their DNA's extraction and also an amplification of region TEF 1 alpha. The PCR products were sequenced and compared with the NCBI database and

1 Recibido: 8 de diciembre, 2016. Aceptado: 14 de marzo, 2017. Este trabajo formó parte de la Tesis de Pregrado del primer autor. Ingeniería Agronómica. Universidad Nacional de Colombia, Sede Palmira, Colombia.

2 Universidad Nacional de Colombia, Sede Palmira, Facultad de Ciencias Agropecuarias, Grupo de Investigación Protección Vegetal para el Mejoramiento de la Productividad. Colombia. edhenaoh@unal.edu.co, hernandezcarlos887@gmail.com, martha474@gmail.com, eydgomezlo@unal.edu.co

3 Universidad de Nariño, Facultad de Ciencias Agrícolas. Pasto, Nariño, Colombia. claudiasalazarg@yahoo.com

4 Federación Nacional de Cafeteros de Colombia. Extensionista. Edwin.Henao@cafedecolombia.com.co
\end{abstract}


Fusarium ID. The results obtained by sequencing showed that the isolates belong to Fusarium incarnatum, Fusarium solani, and Fusarium proliferatum, the first species had a mayor occurrence in the tested samples. The analysis of phylogenetic relationships was achieved by using the 6 MEGA program, and by using the similarity coefficient nearest neighbor, which allowed to observe the variability among the evaluated isolates.

Keywords: fusariosis, Passiflora edulis, fungal diseases, genetic variation.

\section{Introducción}

La producción de maracuyá en Colombia es prometedora y ocupa los primeros puestos en el mundo; lo anterior, debido a las características organolépticas y nutritivas que presentan las diferentes variedades (Cubillos et al., 2011). Esta fruta fue introducida en el país a inicios de la década de 1960 y, hasta el 2012 existían cerca de 5800 hectáreas con una producción de 90000 toneladas, de las cuales el 65\% son destinadas a la industria para jugos concentrados de exportación (Ocampo et al., 2013). No obstante, esta producción se ve afectada por problemas fitosanitarios ocasionados por diversos microorganismos, entre los que se encuentran hongos saprófitos, endófitos o patogénicos del género Fusarium, los cuales pueden ser causantes de síntomas como marchitez y amarillamiento de las hojas, seguido de secamiento de las ramas, fuerte defoliación e incluso la muerte de la planta (Gómez-López et al., 2014). Diversas especies de Fusarium se han reportado como patógenos de numerosos cultivos, ocasionando pérdidas leves hasta el total de la producción. Como en frijol, donde se han encontrado pérdidas del 100\% cuando los cultivares utilizados son susceptibles y las condiciones ambientales son favorables al patógeno (Parry et al., 1995; Dean et al., 2012; Perniola et al., 2014). Específicamente en el cultivo de maracuyá, Fusarium oxysporum f. $s p$. passiflorae se ha manifestado como agente causal de la marchitez vascular, secadera o fusariosis provocando una disminución del rendimiento entre 40-60\% (Martínez-Bolaños, 2012), por lo que, es importante generar diagnósticos de las especies asociadas.

En maracuyá, el género Fusarium fue reportado por primera vez en Australia en el año 1951 (Fischer y Rezende, 2008), y se ha definido como un hongo con actividad enzimática celulolítica que le permite establecerse en la superficie de la planta, constituida principalmente de celulosa; lo que produce ablandamiento por la desintegración de los componentes de la pared celular (Agrios, 2005; Valencia-Guerrero et al., 2011); es decir, permite la degradación de barreras de protección y compuestos intercelulares de las plantas. Estas características lo ubican como uno de los más importantes patógenos del mundo agrícola (Kikot, 2012).

Desde el descubrimiento del ADN, desarrollo de la biología molecular y el avance en el entendimiento de las relaciones filogenéticas entre algunos organismos, se han desarrollado diversas técnicas moleculares que permiten un rápido y confiable diagnóstico de microorganismos, además, contribuyen a la identificación de especies, entre las que se encuentran las del género Fusarium. Este género ha presentado una clasificación taxonómica ampliamente discutida, teniendo en cuenta que los caracteres morfológicos, en muchos casos, resultan ser insuficientes para una correcta identificación a nivel de especie, por lo que, se ha hecho necesaria la implementación de estas técnicas moleculares. Entre las técnicas moleculares se tiene la comparación de pequeñas secuencias de ADN de regiones que son altamente conservadas como la calmodulina, $\beta$ tubulina, la histona H3, ITS y TEF1- $\alpha$. Así mismo, el gen TEF1- $\alpha$ o factor de elongación de la traducción es una de las regiones más empleadas en la identificación molecular de hongos, entre los que se encuentra Fusarium, ya que muestra un alto nivel de polimorfismo entre especies estrechamente relacionadas (O’Donnell et al., 1998; Lesli et al., 2006; Gómez-López, 2008; Aoki et al., 2014).

El objetivo de esta investigación fue identificar molecularmente las poblaciones de Fusarium presentes en maracuyá (Passiflora edulis). 


\section{Materiales y métodos}

\section{Localización geográfica}

Esta investigación fue ejecutada en el periodo comprendido entre enero y diciembre de 2015. Los aislamientos de Fusarium se obtuvieron de muestras vegetales (raíz, tallo, hojas, flores y frutos) con síntomas de marchitez o fusariosis. Luego de realizar un recorrido e inspección a todas las plantas de cultivos de maracuyá (Passiflora edulis) ubicados en cinco localidades del Valle del Cauca (Cuadro 1), las muestras fueron colectadas y empacadas en bolsas de papel y plástico para ser llevadas al laboratorio de diagnóstico vegetal de la Universidad Nacional de Colombia, sede Palmira (1050 msnm; $23{ }^{\circ} \mathrm{C}$ y $70 \%$ de humedad relativa), en donde fueron procesadas.

Cuadro 1. Georeferenciación de las muestras de maracuyá (Passiflora edulis) colectadas en el Valle del Cauca, Colombia. Abril 2015. Table 1. Passion fruit samples georeferencing (Passiflora edulis) collected in Valle del Cauca, Colombia. April, 2015.

\begin{tabular}{|c|c|c|c|c|c|}
\hline Codigo aislmiento & Latitud & Longitud & Msnm & Municipio & Finca \\
\hline FmTruf1 & $4^{\circ} 12^{\prime} 27,2^{\prime \prime} \mathrm{N}$ & $76^{\circ} 19^{\prime} 08,7^{\prime \prime} \mathrm{W}$ & 1260 & Trujillo & La Juana \\
\hline FmAndf2 & $4^{\circ} 09^{\prime} 54,2^{\prime \prime} \mathrm{N}$ & $76^{\circ} 09^{\prime} 51,5^{\prime \prime} \mathrm{W}$ & 995 & Andalucía & El Encanto \\
\hline FmBolf3 & $4^{\circ} 16^{\prime} 19,7^{\prime \prime} \mathrm{N}$ & $76^{\circ} 12^{\prime} 37,6^{\prime \prime} \mathrm{W}$ & 978 & Bolívar & La Herradura \\
\hline FmJamf4 & $3^{\circ} 07^{\prime} 48,7^{\prime \prime} \mathrm{N}$ & $76^{\circ} 35^{\prime} 44,1^{\prime \prime} \mathrm{W}$ & 869 & Jamundí & Robles \\
\hline FmTorf5 & $4^{\circ} 37^{\prime} 04,8^{\prime \prime} \mathrm{N}$ & $76^{\circ} 05^{\prime} 39,4^{\prime \prime} \mathrm{W}$ & 960 & Toro & Castellana \\
\hline FmBolf6 & $4^{\circ} 16^{\prime} 35,0^{\prime \prime} \mathrm{N}$ & $76^{\circ} 12^{\prime} 58,3^{\prime \prime} \mathrm{W}$ & 968 & Bolívar & La Herradura \\
\hline FmBolf7 & $4^{\circ} 16^{\prime} 19,5^{\prime \prime} \mathrm{N}$ & $76^{\circ} 12^{\prime} 34,7 ’ \mathrm{~W}$ & 978 & Bolivar & La Herradura \\
\hline FmBolh8 & $4^{\circ} 16^{\prime} 18,4^{\prime \prime} \mathrm{N}$ & $76^{\circ} 12^{\prime} 34,0^{\prime \prime} \mathrm{W}$ & 978 & Bolivar & La Herradura \\
\hline
\end{tabular}

Código: F (Fusarium); m (Maracuyá); Tru (Trujillo); And (Andalucía); Bol (Bolívar); Jam (Jamundî); Tor (Toro); f (Fruto); h (Hojas) / Code: F (Fusarium); m (Passion fruit); Tru (Trujillo); And (Andalucía); Bol (Bolívar); Jam (Jamundí); Tor (Toro); f (Fruit); h (Leaves).

\section{Aislamiento de hongos del género Fusarium}

Para aislar el Fusarium spp. que se encontraba asociado a maracuyá, se procesaron un total de 35 muestras vegetales provenientes de las cinco localidades de estudio. Posteriormente, en el laboratorio de diagnóstico vegetal se procedió a realizar un lavado del material con Twin 20, luego se cortaron trozos de cinco mm del órgano afectado (raíz, tallo, hojas, flores o frutos) para su posterior lavado y desinfección con alcohol al 70\%, hipoclorito 1\% y agua destilada estéril; sumergiéndolo por un minuto en cada solución (Carrillo, 2003; Henao et al., 2015). Luego se empleó el medio de cultivo papa dextrosa agar (PDA), según las características del género (Gómez-López, 2008), a razón de 39 g/l; se colocó el tejido desinfectado en cajas de petri con PDA que previamente se había dejado secar en cámara de flujo laminar, para evitar crecimiento bacteriano por exceso de humedad. Una vez sembrados en el medio, se incubaron a $25^{\circ} \mathrm{C}$ durante siete días, con el objetivo de generar condiciones ideales para la propagación del hongo.

\section{Obtención de cultivo puro y monospórico}

De las 35 muestras procesadas se obtuvieron ocho aislamientos de Fusarium spp., los cuales fueron seleccionados de acuerdo con el crecimiento, forma y coloración de la colonia, así como observación de conidias, 
conidióforo y estructuras reproductivas al microscopio. Los aislamientos que crecieron de cada uno de los tejidos sembrados en PDA y que presentaron las características antes mencionadas (ocho en total), fueron replicados en PDA, para obtener cultivo puro y proceder a la obtención de cultivos monospóricos (Cañedo y Ames, 2004), técnica que consiste en generar crecimiento del hongo a partir de una conidia, por medio de diluciones seriadas de una suspensión de conidias hasta $10^{-6}$. De la última dilución se tomarón $100 \mu \mathrm{l}$ y se adicionaron a medio agar agua (AW); luego, se incubó a $25^{\circ} \mathrm{C}$, hasta observar germinación de una conidia que inmediatamente era transferida a PDA. Por último, se incubó a $25^{\circ} \mathrm{C}$ por diez días para realizar la extracción de ADN (Ortu et al., 2013; Hernández et al., 2015a; 2015b; Henao et al., 2015) y asegurar pureza genética (Gómez-López, 2008).

\section{Extracción de ADN}

El micelio del cultivo monospórico fue raspado y depositado en un tubo de Eppendorf estéril de $2 \mathrm{ml}$, y la extracción de ADN se realizó con base en los protocolos de Wilson (1987) con algunas modificaciones de Gómez-López (2008). Posteriormente, se realizó la reacción en cadena de la polimerasa (PCR) con base en los planteamientos de O’Donnell et al. (1998) y Hernández et al. (2015b). Se amplificó el factor de elongación de la traducción (TEF 1 alpha) usando los cebadores Ef1 $\alpha$ (5'-ATGGGT AAGGAAG ACAAGAC-3') y Ef2 (5'-GGAAG TACC AGT GAT CATGTT-3'). Se preparó una solución final de $25 \mu$, que contenía $12,5 \mu l$ de DreamTaq Green PCR Master Mix (1,5 mM MgCl$; 200 \mathrm{nM}$ dNTPs), $0,5 \mu l$ del iniciador 1 (10 pmol/ $\mu \mathrm{l}) ; 0,5 \mu \mathrm{l}$ del iniciador $2(10 \mathrm{pmol} / \mu \mathrm{l}) ; 9,5 \mu \mathrm{l}$ de agua ultra pura y $2 \mu \mathrm{lde} \operatorname{ADN}(20 \mathrm{ng} / \mu \mathrm{l})$. El control positivo fue cedido por el laboratorio de diagnóstico vegetal, mientras el control negativo estaba constituido por el coctel para PCR sin la adición de ADN. La PCR se realizó en un termociclador bajo las siguientes condiciones: desnaturalización inicial a $95{ }^{\circ} \mathrm{C}$ por $120 \mathrm{~s}$ con 35 ciclos; segunda desnaturalización a $95^{\circ} \mathrm{C}$ por $30 \mathrm{~s}$; hibridación a $57{ }^{\circ} \mathrm{C}$ por $30 \mathrm{~s}$; extensión de $\mathrm{ADN}$ a $72{ }^{\circ} \mathrm{C}$ por $60 \mathrm{~s}$; extensión final de $\mathrm{ADN}$ a $72{ }^{\circ} \mathrm{C}$ por $10 \mathrm{~min}$.

Por electroforesis, el producto de PCR se visualizó en gel de agarosa al 1\% a 65 voltios por 90 min. Se usó un marcador de peso GeneRuler 50 pb DNA Ladder; lo que permitió observar fragmentos entre 600 a 700 pb. Los productos de PCR fueron enviados a secuenciar a Macrogen, Corea. La alineación de las secuencias se realizó por ClustaW y el árbol filogenético se construyó con el método del "vecino más cercano" (neighbor joining tree) (Lin et al., 2014) con el empleo de los programas BioEdit 7.2.5 y Mega 6 (Henao et al., 2015). La secuencia de Clonostachys rosea (Accesión $\mathrm{N}^{\circ}$ KJ654524.1) depositada en la base de datos del GenBank, fue seleccionada como especie referencia o para la construcción del árbol filogenético.

Para determinar la identidad genética o la especie de los ocho aislamientos, las secuencias fueron analizadas y comparadas en las bases de datos del NCBI (National Center for Biotecnology Information) por BLAST (Basic Local Alignment Search Tool) y en la base de datos Fusarium ID.

\section{Resultados}

De las 35 muestras de tejido vegetal provenientes de cinco municipios del Valle del Cauca, Colombia, se obtuvieron ocho aislamientos del género Fusarium, de los cuales, siete fueron encontrados asociados a frutos y el restante a hoja. No se encontraron aislamientos en tallo, raíz o flores, entre las muestras evaluadas, aun cuando se utilizó el mismo muestreo en las diferentes localidades y para los diversos tejidos vegetales, al recorrer e inspeccionar la totalidad de las plantas y partes de las mismas, con síntomas de enfermedad. La caracterización molecular de los ocho aislamientos permitió identificar tres especies: Fusarium incarnatum, Fusarium proliferatum y Fusarium solani; la primera fue la de mayor ocurrencia en las muestras evaluadas (Cuadro 2). 
Cuadro 2. Número de accesión y porcentaje de similitud (según GenBank) de aislamientos de Fusarium identificados por secuenciación con el marcador TEF 1 alpha aislados de maracuyá (Passiflora edulis), en el Valle del Cauca, Colombia. Agosto. 2015.

Table 2. Accession number and percentage of similarity (as GenBank) of Fusarium isolates identified by sequencing with the TEF 1 alpha marker isolated from passion fruit (Passiflora edulis), in Valle del Cauca, Colombia. August, 2015.

\begin{tabular}{llcc}
\hline Código & TEF 1 alpha & Accesión & Similitud \% \\
\hline FmTruf1 & F. incarnatum & KF993969.1 & 99 \\
FmAndf2 & F. incarnatum & JX268996.1 & 99 \\
FmBolf3 & F. proliferatum & KF575335.1 & 99 \\
FmJamf4 & F. incarnatum & KF993969.1 & 99 \\
FmTorf5 & F. incarnatum & JX268996.1 & 99 \\
FmBolf6 & F. proliferatum & KT211614.1 & 99 \\
FmBolf7 & F. solani & KT884105.1 & 99 \\
FmBolh8 & F. incarnatum & JX268996.1 & 98 \\
\hline
\end{tabular}

\section{Relaciones filogenéticas}

Se obtuvieron ampliaciones entre 600 y 700 pb aproximadamente. La construcción del árbol filogenético, permitió agrupar las ocho secuencias encontradas en tres grupos o clados de acuerdo con la homología en las secuencias, y dejó como clado externo la secuencia de Clonostachys rosea (Accesión KJ654524.1) utilizada como secuencia de referencia o raíz.

\section{Discusión}

Lo hallado en esta investigación coincide con Fischer et al. (2007), Campo-Arana y Vergara-Canedo (2014), quienes reportaron a $F$. proliferatum y $F$. solani asociados al cultivo de maracuyá. No obstante, $F$. incarnatum, no se encontró reportado como microorganismo asociado al cultivo de estudio, es por ello que, los resultados de esta investigación son un nuevo reporte para este cultivo y para la especie de hongo, $F$. incarnatum se ha descrito como un hongo principalmente saprófito y productor de micotoxinas en diferentes plantas (Li et al., 2008; Pérez et al., 2012; Castellá y Cabañes, 2014); sin embargo, en estudios recientes se ha venido reportando como un hongo patógeno que ataca a especies vegetales como Capsicum spp., el nogal, entre otras (Seta et al., 2004; Singh et al., 2011; Ramdial et al., 2016).

El gen TEF 1 alpha (TEF1 $\alpha$ ) permitió obtener unos amplicones dentro del tamaño esperado y reportado (O'Donnell et al., 1998). Las secuencias de los ocho aislamientos del género Fusarium, se agruparon en tres clados o grupos, de acuerdo con la homología en las secuencias, probablemente debido a que parten de un ancestro común (Geisser et al., 2013). El primer clado incluyó los aislamientos FmTruf1, FmJamf4, FmBolh8, FmAndf2 y FmTorf5, los cuales presentaron secuencias con un porcentaje de similitud entre el $98 \%$ a $99 \%$ con las secuencias registradas en el GenBank como $F$. incarnatum. El segundo clado, F. proliferatum; agrupado por los aislamientos FmBolf3 y FmBolf6. Y el último, F. solani, asociado al aislamiento FmBolf7 (Figura 1; Cuadro 2). Esta agrupación por clados se asemeja a la propuesta por Aoki et al. (2014) en cuanto a la distancia y organización entre clados; 




Figura 1. Árbol filogenético y agrupación por clados de las secuencias de Fusarium con el primer TEF 1 alpha, con raíz de $C$. rosea . Febrero, 2016.

Figure 1. Phylogenetic tree grouping of Fusarium clades and sequences with primer TEF 1 alpha, with C. rosea root. February, 2016.

ya que, en el árbol filogenético que estos autores presentaron, el clado $F$. incarnatum-equiseti, al que pertenece $F$. incarnatum se encuentra más emparentado o genéticamente más próximo al clado $F$. fujikuroi, al que hace parte $F$. proliferatum; mientras que, se encuentra más lejano y en un grupo diferente a $F$. solani.

La variabilidad entre las especies que se muestra en el árbol filogenético, pudo deberse a que los diferentes aislamientos pertenecían a un origen filogenético o linaje diferente (Nelson et al., 1994; Edel et al., 2001; Leslie et al., 2006; O’Donnell et al., 2008; Balajee et al., 2009; Salazar-González et al., 2016).

Las secuencias permitieron observar una variabilidad intraespecífica, sobre todo en el clado o grupo de $F$. proliferatum, la cual puede tener explicación en la relación entre los caracteres genotípicos y la localización geográfica del hospedante del que fueron aisladas. Aunque ambas muestras provenían del mismo municipio y tipo de tejido (fruto), las localidades o fincas, de donde fueron tomadas las muestras y aislados los hongos, son diferentes. Por otro lado, dentro de los complejos se ha documentado la existencia de grandes diferencias genéticas, derivadas de un origen filogenético diferente (Nelson et al., 1993; O’Donnell et al., 2008; Balajee et al., 2009; Aoki et al., 2014).

Las especies obtenidas mediante el uso gen TEF 1 alpha, demostraron la eficiencia del mismo y su utilidad para identificar el polimorfismo en este género; debido a que como lo plantean Wulff et al. (2010) y Salazar-González et al. (2016), tiene alto grado de polimorfismo e identifica una fracción única del gen TEF $1 \alpha$ relacionado con la traducción proteica.

\section{Conclusiones}

Fusarium incarnatum, Fusarium proliferatum y Fusarium solani se encontraron asociados a maracuyá en el Valle del Cauca, y los resultados obtenidos mostraron mayor predominancia de la especie $F$. incarnatum. 
El uso de métodos moleculares permitió obtener información filogenética y taxonómica de los aislamientos y las especies de Fusarium asociadas a maracuyá, y reafirma que la PCR acompañada de la amplificación del genTEF 1 alpha, es adecuada para la identificación de especies de este género.

El análisis de las relaciones filogenéticas reveló variabilidad intraespecífica entre los aislamientos evaluados, que están asociados a hojas y frutos, teniendo mayor presencia en frutos, lo que implica generar nuevas investigaciones y soluciones al potencial daño en cosecha y poscosecha que puede disminuir el valor comercial del maracuyá, como también, la relación del microorganismo con la generación de metabolitos complementarios como las micotoxinas, que pueden afectar al ser humano.

\section{Agradecimientos}

A la Universidad Nacional de Colombia sede Palmira. A la Convocatoria HERMES 2013-2015. Al Laboratorio de Diagnóstico Vegetal por prestar sus instalaciones. Al grupo de Investigación Protección Vegetal para el Mejoramiento de la Productividad.

\section{Literatura citada}

Agrios, G. 2005. Plant pathology. $5^{\text {th }}$ ed. Elsevier Academic Press, San Diego, CA, USA.

Aoki, T., K. O’Donnell, and D.M. Geiser. 2014. Systematics of key phytopathogenic Fusarium species: current status and future challenges. J. Gen. Plant Pathol. 80:189-201. doi:10.1007/s10327-014-0509-3

Balajee, S.A., A.M. Borman, M.E. Brandt, J. Cano, M. Cuenca-Estrella, E. Dannaoui, J. Guarro, G. Haase, C.C. Kibbler, W. Meyer, K. O’Donnell, C.A. Petti, J.L. Rodriguez-Tudela, D. Sutton, A. Velegraki, and B.L Wickes. 2009. Sequence-based identification of Aspergillus, Fusarium, and Mucorales species in the clinical mycology laboratory: where are we and where should we go from here? J. Clin. Microbiol. 47:877-884. doi:10.1128/JCM.01685-08

Campo-Arana, O., y L. Vergara-Canedo. 2014. Evaluación de la resistencia de la población de maracuyá amarillo (Passiflora edulis f. flavicarpa. Degener), de San Isidro, Córdoba, a la marchitez vascular (Fusarium spp.). Fitopatol. Colomb. 38:39-42.

Cañedo, V., y T. Ames. 2004. Manual de laboratorio para el manejo de hongos entomopatógenos. Centro Internacional de la Papa (CIP), PER. http://www.cipotato.org/library/pdfdocs/AN65216.pdf (consultado 15 may. 2016).

Carrillo, L. 2003. Los hongos de los alimentos y los forrajes. Universidad Nacional de Salta, ARG. http://www.microbiota.com. ar/sites/default/files/0cubierta.pdf (consultado 23 nov. 2016).

Castellá, G., and F.J. Cabañes. 2014. Phylogenetic diversity of Fusarium incarnatum-equiseti species complex isolated from Spanish wheat. Antonie Van Leeuwenhoek 106:309-317. doi:10.1007/s10482-014-0200-x

Cubillos, J.G., A. Páez, y L. Mejía. 2011. Evaluación de la capacidad biocontroladora de Trichoderma harzianum Rifai contra Fusarium solani (Mart.) Sacc. asociado al complejo "Secadera” en Maracuyá, bajo condiciones de invernadero. Rev. Fac. Nal. Agron. Medellín. 64:5821-5830.

Dean, R., J.A. Van-Kan, Z.A. Pretorius, K.E. Hammond-Kosack, A. Di-Pietro, P.D. Spanu, J.J Rudd, M. Dickman, R. kahmann, J. Ellis, and G.D. Foster. 2012. The Top 10 fungal pathogens in molecular plant pathology. Mol. Plant Pathol. 13:414-430. doi:10.1111/j.1364-3703.2011.00783.x

Edel, V., N. Steinberg, G. Gautheron, C. Recorbet, and C.Alabouvette. 2001. Genetic diversity of Fusarium oxysporum populations isolated from different soils in France. FEMS Microbiol. Ecol. 36:61-71. doi:10.1146/annurev.phyto.40.030402.110010. 
Fischer, I.H., M. de-Arruda, A. de-Almeida, M.J. García, E. Jeronimo, R. Nakazato, e R. de-Almeida. 2007. Doenças e características físicas e químicas pós-colheita em maracujá amarelo de cultivo convencional e orgânico no centro oeste paulista. Rev. Bras. Frutic. 29:254-259. doi:10.1590/S0100-29452007000200013

Fischer, I., and J. Rezende. 2008. Diseases of passion flower (Passiflora spp.). Pest Technol. 2:1-19.

Geiser, D.M., T. Aoki, C.W. Bacon, S.E. Baker, M.K. Bhattacharyya, M.E. Brandt, D.W. Brown, L.W. Burgess, S. Chulze, J.J. Coleman, J.C. Correll, S.F. Covert, P.W. Crous, C.A. Cuamo, G.S. De-Hoog, A. Di-Pietro, W.H. Elmer, L. Epstein, R.J. Frandsen, S. Freeman, T. Gagkaeva, A.E. Glenn, T.T. Gordon, N.F. Gregory, K.E. Hammond-Kosack, L.E. Hanson, M.M. Jímenez-Gasco, S. Kang, H.C. Kistier, G.A. Kuldau, J.F. Leslie, A. Logrieco, G. Lu, E. Lysøe, L.J. Ma, S.P. McCormick, Q. Migheli, A. Moretti, F. Munaut, K. O`Donnell, L. Pfenning, R.C. Ploetz, R.H. Proctor, S.A. Rehner, V.A. Robert, A.P. Rooney, B. Bin-Salleh, M.M. Scandiani, J. Scauflaire, D.P. Short, E. Steenkamp, H. Suga, B.A. Summerell, D.A. Sutton, U. Thrane, F. Trail, A. Van-Diepeningen, H.D. Vanetten, A. Vijoen, C. Waalwijk, T.J. Ward, M.J. Wingfield, J.R. Xu, X.B. Yaang, T. Yli-Mattila, and N. Zhang. 2013. One fungus, one name: defining the genus Fusarium in a scientifically robust way that preserves longstanding use. Phytopathol. 103:400-408. doi:10.1094/PHYTO-07-12-0150-LE

Gómez-López, E.D. 2008. Caracterización de cepas toxigénicas del género Fusarium mediante técnicas de biología molecular. Tesis Doc., Universidad Politécnica de Valencia, ESP.

Gómez-López, E.D., N.C. Mesa, C.A. Hernández-M, Y.M. Mena, K. Imbachi, J.A. Salazar, S. Benítez, R. López, E.I. Estrada, R. Reina, E.D. Henao, O.E. Sánchez, Y. García, C.A. Huertas, I. Rodríguez, G.M. Cobo, M.L. Velasco, D. Vásquez, E.Y. Sánchez, P. Ramos, Y. Peteche, Y. Piza, S. Rodríguez, E.P. Barinas, L. Álvarez, C. Vega, H. Marulanda, L.A. Londoño, O. Sánchez, P.E Gamboa, y R. Romero. 2014. Programa de manejo fitosanitario en el maracuyá en el Departamento del Valle del Cauca. Universidad Nacional de Colombia, COL.

Henao, E.D., E.D. Gómez, C.A. Hernández, C. Salazar, y M.L. Velasco. 2015. Caracterización molecular de aislamientos de hongos del género Fusarium asociados a aguacate, en el Valle del Cauca-Colombia. En: Sociedad Chilena de Fitopatología, editores, Memorias XXIV Congreso de la Sociedad Chilena de Fitopatología. Viña del Mar, CHI. p. 32.

Hernández, C.A., E.D. Gómez, E.D. Henao, M.L. Velasco, and S. Rodríguez. 2015a. Molecular characterization of Fusarium isolates associated with fruit-bearing plants in Valle del Cauca, Colombia. ASCOLFI 39(1):11.

Hernández, C.A., E.D. Henao, M.L. Velasco, y E.D. Gómez. 2015b. Caracterización morfológica y molecular de aislados de Fusarium asociadas aguacate (Persea americana Mill), en el Valle del Cauca. Fitopatol. Colomb. 39:51-56.

Kikot, G. 2012. Caracterización bioquímica, fenotípica y molecular de aislamientos de Fusarium graminearum provenientes de la región pampeana en relación a la patogenicidad. Tesis Doc., Universidad Nacional de La Plata, Buenos Aires, ARG. http://sedici.unlp.edu.ar/handle/10915/18266 (consultado jul. 2016).

Leslie, J.F., B.A. Summerell, and S. Bullock. 2006. The Fusarium laboratory manual. Blackwell Publishing, Ames, IA, USA.

Li, L.Y., Y. Ding, I. Groth, K.D. Menzel, G. Peschel, K. Voigt, Z.W. Deng, I. Sattler, and W.H. Lin. 2008. Pyrrole and indole alkaloids from an endophytic Fusarium incarnatum (HKI00504) isolated from the mangrove plant Aegiceras corniculatum. J. Asian Nat. Prod. Res. 10:765-770. doi:10.1080/10286020802031106

Lin, Z., S. Xu, Y. Que, J. Wang, J.C. Comstock, J. Wei, P.H. McCord, B. Chen, R. Chen, and M. Zhang. 2014. Species-specific detection and identification of Fusarium species complex, the causal agent of sugarcane pokkah boeng in China. PloS One 9:e104195. doi:10.1371/journal.pone.0104195.

Martínez-Bolaños, A.M. 2012. Evaluación y selección de cepas de Trichoderma sp. para control biológico de Fusarium sp. en maracuyá (Passiflora edulis, variedad flavicarpa), en condiciones in vitro. Tesis Lic., Universidad Técnica de Ambato, Ambato, ECU. 
Nelson, P.E., A.E. Desjardins, and R.D. Plattner. 1993. Fumonisins, mycotoxins produced by Fusarium species: biology, chemistry, and significance. Ann. Rev. Phytopathol. 31:233-252. doi:10.1146/annurev.py.31.090193.001313

Nelson, P.E., M.C. Dignani, and E.J. Anaissie. 1994. Taxonomy, biology, and clinical aspects of Fusarium species. Clin. Microbiol. Rev. 7:479-504. doi:10.1111/j.1470- 9465.2004.00845.x?

Ocampo, J., R. Urrea, K. Wyckhuys, y M. Salazar. 2013. Exploración de la variabilidad genética del maracuyá (Passiflora edulis f. flavicarpa Degener) como base para un programa de fitomejoramiento en Colombia. Acta Agron. 62:352-360.

O’Donnell K., H.C. Kistler, E. Cigelnik, and R.C. Ploetz. 1998. Multiple evolutionary origins of the fungus causing Panama disease of banana: concordant evidence from nuclear and mitochondrial gene genealogies. Proc. Natl. Acad. Sci. 95: 2044-2049. doi:10.1073/pnas.95.5.2044

O’Donnell, K., D.A. Sutton, A. Fothergill, D. McCarthy, M.G. Rinaldi, M.E. Brandt, N. Zhang, and D.M. Geiser. 2008. Molecular phylogenetic diversity, multilocus haplotype nomenclature, and in vitro antifungal resistance within the Fusarium solani species complex. J. Clin. Microbiol. 46:2477-2490. doi:10.1128/JCM.02371-07

Ortu, G., M.L. Bertetti, M.L. Gullino, and A. Garibaldi. 2013. A new forma specialis of Fusarium oxysporum on Crassula ovata. J. Plant Pathol. 95:33-39. doi:10.4454/JPP.V95I1.003

Parry, D.W., P. Jenkinson, and L. McLeod. 1995. Fusarium ear blight (scab) in small grain cereals - a review. Plant Pathol. 44:207-238. doi:10.1111/j.1365-3059.1995.tb02773.x

Pérez, Y., G. Gómez, M. González, D. Vaillant, E. Ramos, J.R. Palacios, M. Pérez, y J. Almándoz. 2012. Hongos patógenos en plantas ornamentales de importancia para Cuba. Fitosanidad 15:205-214.

Perniola, O.S., S. Staltari, S.E. Chorzempa, M.M. Astiz-Gassó, and M.d.C. Molina. 2014. Control biológico de Fusarium graminearum: utilización de Trichoderma spp. y biofumigación con parte aérea de Brassica juncea. Rev. Fac. Cienc. Agrar. Univ. Nac. Cuyo 46(2):45-56.

Ramdial, H., F. Hosein, and S.N. Rampersad. 2016. First report of Fusarium incarnatum associated with fruit disease of bell peppers in Trinidad. Plant Dis. 100:526-526. doi:10.1094/PDIS-05-15-0550-PDN

Salazar-González, C., L. Serna-Cock, y E. Gómez-López. 2016. Caracterización molecular de Fusarium asociado a pudrición basal del fruto en Pitahaya (Selenicereus megalanthus). Agron. Mesoam. 27:277-285. doi:10.15517/am.v27i2.21269

Seta, S., M. Gonzalez, and G. Lori. 2004. First report of walnut canker caused by Fusarium incarnatum in Argentina. Plant Pathol. 53:248. doi:10.1046/j.1365-3059.2003.00968.x

Singh, B., C.S. Kalha, V.K. Razdan, and V.S. Verma. 2011. First report of walnut canker caused by Fusarium incarnatum from India. Plant Dis. 95:1587-1587. doi:10.1094/PDIS-04-11-0352

Valencia-Guerrero, M., B. QuevedoHidalgo, M. Franco-Correa, H. Diez-Ortega, C.M. Parra-Giraldo, y M.X. RodríguezBocanegra. 2011. Evaluación de actividades enzimáticas de Fusarium spp., aislados de lesiones en humanos, animales y plantas. Universitas Sci. 16:147-159. doi:10.11144/javeriana.SC16-2.aoec

Wilson, K. 1987. Preparation of genomic DNA from bacteria. In: F.M. Ausubel et al., editors, Current protocols in molecular biology. John Wiley and Sons, USA. p. 2.4.1-2.4.5.

Wulff, E.G., J.L. Sørensen, M. Lübeck, K.F. Nielsen, U. Thrane, and J. Torp. 2010. Fusarium spp. Associated with rice Bakanae: ecology, genetic diversity, pathogenicity and toxigenicity. Environ. Microbiol. 12:649-657. doi:10.1111/j.14622920.2009.02105.x 